\title{
Using actors and peers in the clinical education of stuttering: Results from a mixed methods study
}

\author{
Charles D. Hughes', Derek E. Daniels², Erik X. Raj ${ }^{3}$, Alana Ghent ${ }^{4}$ \\ ${ }^{1}$ Baldwin Wallace University, Department of Communication Sciences and Disorders, $\mathrm{OH}_{;}{ }^{2}$ Wayne State University, Department of Communication \\ Sciences and Disorders, MI; ${ }^{3}$ Monmouth University, Department of Speech-Language Pathology, NJ; ${ }^{4}$ Eastern Kentucky University, Department of \\ English, KY, USA
}

Purpose: The use of trained actors in the clinical education of helping professionals has been well-documented. However, little is known about the potential benefit of using actors in the clinical education of speech-language pathology students specific to stuttering. This mixed methods study examined the perceptions of 26 participants related to their experiences with peers and two trained actors in a graduate-level fluency disorders course.

Methods: These experiences took place in the context of a simulated meeting following the review of hypothetical fluency diagnostic data. After the simulations, participants provided quantitative and qualitative responses reflecting on their experiences with a peer and actor in regard to their interpersonal skills, ability to communicate diagnostic information, and the benefit the experience had on their learning.

Results: Quantitative ratings showed that participants felt more confident explaining information related to the nature of stuttering with the actor compared to the peer. Among some of themes which emerged from the qualitative analysis were that participants viewed their experiences with the actors as natural, genuine, and contributing to new insights in their abilities.

Conclusions: The use of actors in the clinical education of stuttering was found to be beneficial to student learning related to their clinical skills.

Keywords: Stuttering, Actors, Education, Clinical

\section{INTRODUCTION}

Actors have been commonly used in the training of various helping professions [1-4]. Yet, they have not been frequently used in the field of speech-language pathology [5]. Although actors are seldom utilized in the clinical education of speech-language pathology students, the importance of actors in these training programs has been highlighted [6-9]. Some of this research has highlighted the importance of appropriate actor training to assure accuracy in their role portrayal with students [9], as well as assuring appropriate judges to evaluate student abilities when interacting with actors [8]. Many benefits exist when actors are incorporated into clinical training and involve the consistency of the experience and ability to reflect on the interaction in a standardized manner [9].

Gregg [5] provides a review of the benefit of using actors in speech-language pathology and in the clinical training of stuttering. He goes on to highlight the work of Zraick $[7,10]$ with actors and adapting aspects of his outline [10] and use of Objective Structured Clinical Examination's (OSCE) to fit the purpose for assessing student skills in

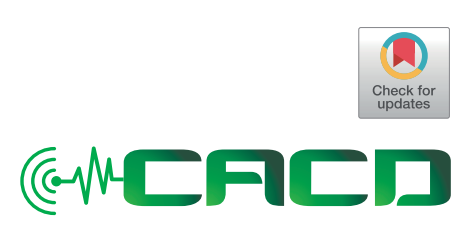

Received: February 22, 2019

Revision: April 3, 2019

Accepted: April 11, 2019

Correspondence:
Charles D. Hughes

Baldwin Wallace University, Department of Communication Sciences and Disorders, 275 Eastland Road, Berea, $\mathrm{OH} 44017$, USA

Tel: $+440-826-5959$

Fax: +440-826-3577

E-mail: chughes@bw.edu

C 2019 The Korean Association of SpeechLanguage Pathologists

This is an Open Access article distributed under the terms of the Creative Commons Attribution NonCommercial License (http://creativecommons.org/ licenses/by-nc/4.0/) which permits unrestricted noncommercial use, distribution, and reproduction in any medium, provided the original work is properly cited. 
working with parents of children who stutter during a diagnostic interview. He goes on to provide suggestions and a framework for the inclusion of actors in a graduate-level course on fluency disorders with the description of biographical sketches of different family members and scenarios related to their reaction to the diagnosis of stuttering. Although others have examined the use and benefit of actors in speech-language pathology $[6,7,10,11,12]$, very little attention has been given to the use and benefit of actors specifically in the clinical training of fluency disorders.

In addition, little is known regarding the difference between student perceptions using peers as simulated clients compared to actors. The use of classmates taking on the role of a client is perhaps one of convenience, as finding actual clients or actors may be difficult to incorporate. Syder [12] was one of the first to formally report on incorporating actors in clinical education for speech-language pathology. Syder [12] acknowledged the limitations in having a classmate portray a simulated client, as a classmate's performance may not be authentic. Therefore, Syder [12] used two amateur, paid actors in a clinical skills course with two groups of students, which consisted of first and second-year master's students in speechlanguage pathology. The two actors took on the role of a client with vocal abuse symptoms and a person who stutters. Students participated in individual and group session with the actors. During the group session, a "time out" procedure was used where a student could ask for feedback and support from classmates and the instructor, and reflect on the clinical interaction with the actor as they moved forward with the simulated interaction. Syder [12] asked students to reflect on their experience with actors and used a survey with three open-ended questions. Results suggested that students found the experience beneficial. Differences emerged between first and second year students with one notable difference being that second year students found the individual sessions with the actors more beneficial than the group sessions. Syder [12] recommended that scales needed to be developed that measure student performance with actors. Furthermore, Syder [12] indicated actors could be used in the context of an assessment with parents.

It is important to understand the difference between student experiences with a peer compared to an actor, to determine how effective and authentic the use of both these individuals are in the clinical education experience. Previous research with clinical simulations have focused on client-clinician interactions, however; very little research has been di- rected with parent-clinician interactions during simulations. Furthermore, parent-clinician communications occur frequently in the practice of speech-language pathology, especially when working with preschoolers who stutter, where there is general agreement on the importance of communicating clinical information about stuttering to parents of preschoolers [5,13].

The purpose of this study was to examine the impact of incorporating trained actors in a graduate-level fluency disorders course within the context of a course assignment designed to assess communicating clinical information from a hypothetical case-study. Furthermore, this study was designed to understand the potential differences in student selfperceptions of clinical skills using role-playing with a peer compared to an actor for the assignment. In order to understand the complexity of this interaction, a mixed-methods approach was utilized.

\section{METHODS}

The following sections outline the methodology incorporated in the current study. This section first describes the participants and the class assignment which was analyzed. Due to the assignment involving the use of actors, a description of how the actors prepared for their role is outlined. Finally, information related to the self-assessment scales used is further described along with how the qualitative and quantitative data were analyzed.

\section{Participants}

Twenty-six of the 29 students enrolled in the courses provided their written consent to include their assignment data in the study. Upon reviewing the completed assignments, one participant did not provide a rating for one of the quantitative questions, however, this participant had completed all other questions and it was determined to include this participant in the study. Therefore, 26 participants were included in the study. Participants engaged in an interaction with a trained actor as part of an assignment within a graduate fluency disorders course consisting of two parts with students first interacting with a peer in class, and then with an actor. The assignment was part of a required class project entitled "Clinical Video Samples Project."

\section{Assignment instructions}

Students and actors provided both their written informed 
consent to take part in the study. For this first part of the assignment, participants were required to have a partner, where each took turns taking on the role of being both a clinician and parent of a preschooler with speech fluency concerns. When taking on the role of clinician, participants were instructed to use hypothetical data from a case-history form and disfluency analysis provided by the instructor (first author) to help guide their conversations with their partner, who was taking the role of the parent. The hypothetical assignment data is further described in the following section. Students were required to explain the following information related to stuttering: incidence and prevalence information; gender ratio; variability information; explanations about spontaneous recovery compared to persistent stuttering; and answer questions appropriately related to stuttering from their partner.

In addition to these explanations, students were required to interpret the assessment data and communicate the findings to their peer (assuming the role of the mother). In their conversations, they were required to communicate the following: modifications to the home environment; rationale for any modifications; and to answer at least three questions from their partners related to diagnosis and recommendations. Because of this need to answer questions, partners needed to plan to ask these questions during the interaction. The student pairs were then instructed to schedule a time to meet and record their clinical video. "The video was to be recorded at the university clinic and submitted to the first author after completion. Students were instructed to treat the interaction as if it were a real meeting with a parent. Students were provided with an outline to guide them in their clinical discussions, which was an abbreviated version of the quantitative rating scale they completed for their self-evaluation (see Table 1 for questions)."

\section{Hypothetical assignment data}

As part of the assignment, students were provided with hypothetical assessment data from a preschooler who had suspected fluency concerns. The assessment data consisted of a completed case-history form and disfluency analysis results from two speaking samples, one with the parent and one with

Table 1. Descriptive statistics for peer and actor self-assessment items ( $N=26)$

\begin{tabular}{|c|c|c|}
\hline Section items & $\begin{array}{c}\text { Peer } \\
M d n(I Q R)\end{array}$ & $\begin{array}{c}\text { Actor } \\
M d n(I Q R)\end{array}$ \\
\hline \multicolumn{3}{|l|}{ Interpersonal skills } \\
\hline I Introduced myself in a professional, appropriate manner & $5.00(1.00)$ & $4.00(1.00)$ \\
\hline I maintained appropriate eye contact & $4.00(1.00)$ & $4.00(0.00)$ \\
\hline I used appropriate speech and language & $4.00(0.00)$ & $4.00(1.00)^{\dagger}$ \\
\hline I reacted appropriately to parent/client emotions & $4.00(1.00)$ & $4.00(0.50)$ \\
\hline Total score & $17.00(2.00)$ & $17.00(2.50)^{\dagger}$ \\
\hline \multicolumn{3}{|l|}{ Nature of stuttering } \\
\hline I explained incidence and prevalence of stuttering appropriately for this case & $4.00(0.00)$ & $4.00(1.00)$ \\
\hline I explained the gender ratio of stuttering appropriately for this case & $4.00(0.50)$ & $5.00(1.00)^{*}$ \\
\hline I explained the variability of stuttering appropriately for this case & $4.00(0.50)$ & $4.00(0.00)$ \\
\hline I explained spontaneous recovery and persistent stuttering appropriately & $4.00(0.00)$ & $4.00(1.00)$ \\
\hline I effectively answered questions related to the nature of stuttering & $4.00(0.00)$ & $4.00(1.00)$ \\
\hline Total score & $20.00(2.00)$ & $21.00(3.00)^{*}$ \\
\hline \multicolumn{3}{|l|}{ Diagnostic interpretation and recommendations } \\
\hline I explained the diagnosis of stuttering appropriately & $4.00(0.00)$ & $4.00(0.50)$ \\
\hline I explained modifications to the home environment appropriately & $5.00(1.00)$ & $5.00(1.00)$ \\
\hline I explained the rationale for modifications to the home environment & $4.00(1.00)$ & $4.00(1.00)$ \\
\hline I effectively answered questions related to the diagnosis and recommendations & $4.00(1.00)$ & $4.00(1.00)$ \\
\hline Total score & $16.00(2.50)$ & $17.00(3.00)$ \\
\hline Overall total & $54.00(4.00)$ & $56.00(6.50)^{\dagger}$ \\
\hline
\end{tabular}

${ }^{*} p<0.05 ;{ }^{\dagger} n=25$. 
the clinician. To simulate the experience of reviewing an actual case-history form, the case-history form was completed in handwriting, and then scanned for participants to review. The disfluency analysis samples presented findings related to percentages of total, normal, and stuttering-like disfluencies, as well as comments regarding secondary behaviors.

\section{Self-rating and reflection}

At the conclusion of the peer interaction, students were required to complete a self-assessment of clinical skills (SCS), which consisted of a peer (SCS-P) and actor (SCS-A) version. The SCS was designed for the purposes of the current study and was influenced by and modified from existing literature of clinical simulations in speech-language pathology [5-7,10]. The development of scale items will be further discussed in detail in later sections. The SCS consisted of quantitative ratings of student abilities in regard to their interpersonal skills, nature of stuttering, and diagnostic interpretation and recommendations. Also part of the SCS was a narrative section where students provided further reflection on these three areas (Appendix A), as well as responding to the question of how this experience impacted their learning. The questions from the SCS can be found in Table 1.

\section{Actor version of assignment}

Once students completed their first project with a peer, they then completed the assignment a second time with a trained actor who portrayed the role of a mother of a preschooler with suspected speech fluency concerns. "Due to logistics with coordinating schedules between students and actors, it was determined to be more feasible to have students first meet with peers, then followed by their meetings with the actors." The same instructions and hypothetical assessment data were used with the actor version of the assignment. The first author coordinated the scheduling between each student and one of the two actors used in this project. Students were aware they would be meeting an actor (similar to their peer interaction), and were asked to treat the situation as if it were with a real parent after an assessment. Participants were instructed to meet the actor in the waiting room of the university clinic, and continue to a clinical room where the interaction was video and audio recorded. Following their meeting with the actor, students completed the SCS-A. The only difference between the SCS-A and SCS-P was in relation to the referent of the form, with the SCS-A asking students to reflect on their experience interacting with the actor and communicating the re- quested information about stuttering.

\section{Actors}

\section{Recruitment}

During the previous semester of the fluency disorders course, an online job description was posted advertising open positions for two trained actors. The advertisement went on to state that the actors would be compensated for their time in role preparation and individual performances with students. The description requested that actors be trained in actor research, backstories, and comfortable with improvisation. In this context, the term "improvisation" was included to indicate to potential actors that they would be working from a scenario, and creating characters based on information provided ahead of the exercise. Actors would create dialogue in the moment, while interacting with participants, and in response to the stimulus provided by students playing their roles as clinicians. They would rely on their training in improvisation and their ability to connect with another individual to respond in an immediate way to the dynamic, unscripted situation and to the stimulation supplied by the participants.

The actors' prior training was based in the work of Constantin Stanislaviski, as taught through and described by Benedetti [14], and included developing skills in text analysis, character development, and connection. Skill development included foundational training in using the body, voice, and imagination in the development of character. Text analysis is supported by work in connecting body, voice, and imagination, emphasizing the training techniques of the Chekhov Association [15] and Hagen [16]. In this case "text analysis" meant discovering the broad given circumstance of the situation and their characters, here supplied by the scenario provided by the primary investigator and applying these in preparing their roles. The actors had been trained to apply this information, and their own research, to create a biographical "back story" as a tool to stimulate their imaginations and create authentic characters. In this case, with the guidance of the primary investigator, actors also researched stuttering at a general knowledge level, to add depth and authenticity to their characters.

\section{Demographic information}

As a result of the recruitment efforts, two actors were hired for the project. Both actors are provided with pseudonyms to assure their confidentiality. The first actor, "Jane", was a 22 yearold female who was currently studying Theatre at the university level and had experience acting in multiple roles in the- 
atre productions, as well as directing productions. Jane also reported taking multiple acting classes at the university level and was preparing to finish her degree at the time of the study. "Molly", the second actor, was a 26 year-old female also with formal training from a university. Molly had three years of experience with improvisation, and had multiple professional performances.

\section{Role preparation}

After both actors were hired, they met with the first author to learn how to prepare for their roles and responsibilities related to the course. Similar role preparation approaches have been described by Zraick [10] where actors portraying an individual with aphasia were provided with background notes of the case, specialized feedback and modeling of the desired behaviors for their performance with students. In the current study, since both actors were taking on the role of being the mother to the hypothetical client, the first author followed a similar approach where they provided the actors with foundational information about stuttering that was geared toward the parent's experience. This information consisted of materials provided by the first author, as well as assignments related to their role preparation. Some of these materials provided to actors consisted of an article by Zebrowski and Schum [17], which discussed parental concerns related to stuttering, and how speech-language pathologists can help. In addition, actors and the first author discussed an introductory chapter to stuttering by Guitar [13] to provide actors with characteristics of stuttering. The first author also provided assignments related to role preparation, which were intended to provide opportunities for the actors to prepare for their roles. For example, the first author requested both actors to act "as if" they were someone seeking help for their child who is stuttering and enter a phrase such as "stuttering help" into an internet search engine. This experience helped the actors take the perspective of a parent searching for help for stuttering and what feeling and thoughts a parents might have after reviewing publically available information about help for stuttering on the internet. Actors were encouraged to take notes during this experience and discuss their impressions during weekly meetings with the first author. Another assigned experiential activity consisted of going to the Stuttering Foundation of America's website and listening to the video "Stuttering and Your Child: Help for Parents" [18] and recording their reactions. After viewing the video, actors and the first author discussed their reactions and thoughts. Actors were hired to work approximately 10 hours per week to prepare for their roles and for their performances meeting with students. The role preparation for actors lasted approximately one month with weekly meetings with the first author which were approximately 30 minutes.

In addition to role preparation specific to stuttering, the first author encouraged the actors to utilize their training and education related to this particular role. In developing these characters, actors considered the "Given Circumstances" of the situation in which the characters found themselves, and then supplied their own imaginative details to help bring them to life. Spolin [19] describes given circumstances which includes the "who", "what", "where", and "why" of the situation, as well as the characters' relationship to others present in the room. They considered how elements of spectacle such as props and costumes might help create the realistic details that would allow them to live in the skin of the character and respond as the character might. For instance, one actor gave their character a cell phone. The actor could then invest in the business of being involved in the cell phone rather than in the conversation with the clinician, providing the student clinician an opportunity to experience some of the challenges they might encounter in the field. Actors also costumed their characters in way that was consistent with the character background they had developed.

\section{Biographical sketches}

Toward the conclusion of the actor preparation, both actors developed a biographical sketch for their character in consultation with the first author. This biographical sketch of their character was helpful in approaching their individual performances with students. When the actor met with students, they were encouraged to be spontaneous and to not have a planned script, however, they were asked to remain true to their character. Wherever the student took the conversation, the actors were encouraged to demonstrate improvisation with the given situation. In order to demonstrate the wide variety of parental responses to stuttering, both actors developed different biographical sketches with collaboration and feedback from the first author. Actors used the information they gained through research into stuttering and the given circumstances of the situation, and their biographical sketches to help them make physical and vocal choices that brought the characters to life and enabled them to respond to immediate stimulus in a realistic way. Part of the rationale for having the actors develop two different biographical sketches was 
the fact that students would encounter different personalities and parental reactions to stuttering when working in the field. Furthermore, the use of different backgrounds was also influenced by Gregg [5], who provided a framework for including actors into a graduate fluency disorders course with suggested biographical sketches and guided student self-reflections. Moreover, the background stories for actors were also influenced by and adapted from his suggested framework where he provides profiles of family members of individuals who stutter with varied family dynamic backgrounds which could be used in clinical simulations. Both these biographical sketches are discussed below.

\section{Jane}

This biographical sketch involved a mother who was disengaged from the evaluation process. This actor approached the post-evaluation with an attitude of "I figured I would check it out", and "Someone told me I might want to get this checked out." To illustrate how this parent was disconnected, the actor was distracted throughout the session, oftentimes looking at her cell phone and asking questions like "How long is this going to take?"

\section{Molly}

This actor portrayed a parent that was opposite of the previously mentioned portrayal. This biographical sketch was that of an overly concerned parent. The concern of this parent was communicated in their tone of voice and asking lots of questions. The actor also reported on information they heard about stuttering from the internet.

\section{Influences and development of the SCS}

The motivation for the current study and the development of the SCS were influenced by Gregg [5]. In addition, the SCS items were modified from Zraick [10] and Gregg [5] and adapted for the purposes of the current study. Two items on the interpersonal skills section of the SCS were modified from Zraick's [10] interpersonal skills checklist and consisted of "I introduced myself in a professional, appropriate manner" and "I maintained appropriate eye contact." Furthermore, the Interpersonal Skills, Nature of Stuttering, and Diagnostic Interpretation and Recommendations subsections of the SCS were influenced and modified from Gregg's [5] proposed checklist for measuring student competence during clinical simulations and adapted for the purposes of the study. In addition to the previously mentioned studies, the first author modeled the SCS after course content which was discussed in class related to the nature of stuttering, providing diagnostic information, and therapeutic recommendations, which also were consistent with other rating forms discussed with clinical simulations $[5-7,10]$.

\section{Quantitative data analysis}

Descriptive statistics were calculated for individual items on the SCS-P and SCS-A in the form of means and standard deviations. In addition, a total score for each of three sections was computed (interpersonal skills; nature of stuttering; and diagnostic interpretations and recommendations) for each participant. Means and standard deviations were calculated across participants for each section's total score. Finally, an overall total score was calculated for each participant by adding the total scores for each section. Lastly, a Wilcoxon Sign-Rank Test was performed to compare SCS-P and SCS-A ratings for individual items and total scores across all participants. These descriptive statistics are reported in Table 1.

\section{Qualitative data analysis}

In addition to completing the SCS scales, participants also provided qualitative responses to their experiences. In particular, students were asked to comment on four areas: their interpersonal skills during the interaction, their description of the nature of stuttering, their description of the diagnostic interpretation and recommendations, and the impact of the experience on their learning. Each student completed this for the peer and actor experience. Therefore, a total 52 qualitative responses were reviewed and coded ( 26 responses for the peer interaction and 26 responses for the actor interaction). The constant-comparative method (open, axial, and selective coding) was used to analyze the data [20]. The following steps were used for this process. This procedure has also been used in a previous qualitative study [21].

1. All student, handwritten responses were transcribed and typed verbatim.

2. The first and second author read through all student responses to get a sense of the qualitative data.

3. The first and second author (separately) read through each line of text, and assigned an initial code to each line, or units, of text. A code was considered to be a word or phrase that summarized a line of text, or a unit of text (e.g., two or more related lines of text). This process is referred to as open coding. 
4. Once initial codes were developed individually, the authors began to combine the codes into common categories collaboratively. This process is referred to as axial coding.

5. Once common categories were developed from a list of codes, the categories were arranged into major categories (or themes) and minor categories (or sub-themes). This process is referred to as selective coding.

6. Both authors met to compare findings and discuss how individual codes and categories could be combined into a thematic structure. Agreement was reached on the major and minor themes.

7. When a consensus was reached regarding the thematic structure, a third researcher with experience in qualitative research (third author) was included for the interjudge reliability process. This process involved the first author selecting quotes from each major and minor theme, providing the quotes to the second and third authors, and having all three authors match the quote to a theme. A percentage of agreement was conducted and revealed that all three authors reached $>80 \%$ agreement for all major and minor themes. This process was incorporated to verify the triangulation process between the three researchers to assure reliability.

\section{RESULTS}

\section{Quantitative data}

Results indicated that participants believed they explained the gender ratio related to stuttering significantly better with the actor compared to the peer $(Z=-3.051, p=0.002)$. Furthermore, significant results were found in regard to total scores related to the nature of stuttering $(Z=-3.093, p=0.002)$, with participants having higher ratings with the actor compared to the peer. Although not statistically significant, three other findings are noteworthy to mention. These findings included participants providing higher rating for their interactions with the actor in regard to using appropriate speech and language $(Z=-1.941, p=0.052)$, and explaining the incidence and prevalence of stuttering $(Z=-1.890, p=0.059)$. Lastly, overall total scores comparing peer and actor scores approached the level of statistical significance with $(Z=-1.959, p=0.050)$; with participants' scores being higher for the actor compared to the peer. Table 1 presents descriptive statistics for individual items and total scores on the SCS-P and SCS-A.

\section{Qualitative data}

Thematic analysis was conducted on the retrospective, narrative accounts of student interactions with both a peer and an actor. Through thematic analysis, major and minor themes emerged from the qualitative data. This thematic structure is presented in Table 2. These major and minor themes are discussed in the following sections.

\section{Peer experience reflections Major theme 1: Interpersonal skills}

This theme described the different ways in which participants reflected on their interactions with a peer acting as a parent. These reflections included topics such as establishing rapport, active listening, and using language appropriate to assure their classmate understood their message when communicating about stuttering. In addition, information related to the use of eye contact, reflecting on interpersonal skills, and being empathetic during the interaction were included in this theme category.

Table 2. Thematic structure for peer and actor experiences

\begin{tabular}{cc}
\hline \multicolumn{1}{c}{ Peer } & Major theme 1: interpersonal skills \\
\hline Major theme 1: interpersonal skills & $\bullet$ Minor theme 1: eye contact was good or needed improvement \\
• Minor theme 1: eye contact was good or needed improvement & - Minor theme 2: effective interpersonal skills \\
Major theme 2: clinical conversations & Major theme 2: clinical conversations \\
• Minor theme 3: improvements with explaining stuttering revealed & $\bullet$ Minor theme 3: effective communication about nature of stuttering \\
Major theme 3: impact of the experience & - Minor theme 4: areas of improvement with clinical discussions \\
• Minor theme 5: beneficial learning experience & Major theme 3: impact of experience \\
- Minor theme 6: strengths and weaknesses revealed & - Minor theme 5: natural flow, real-world, and genuine \\
\hline
\end{tabular}


Minor theme 1: Eye contact was good or needed improvement This minor theme referred to participants' descriptions of the manner in which they maintained appropriate eye contact during the interaction. Some participants described their eye contact as effective, while others described it as needing improvement. For example, one participant stated "I believe I did a great job using appropriate speech and language. My eye contact could have been better but I felt more confident once I looked off my notes." Furthermore, another participant statements reflects this theme as they wrote "My ability to maintain eye contact during the conversation is noted as a weakness as I would refer to my notecards to ensure I was covering all requirements of the assignment."

\section{Minor theme 2: Improvements for interpersonal skills}

This theme referred to the participants' reflections on areas of interpersonal skill improvement, such as not interrupting the "parent," maintaining an appropriate posture, and not relying heavily on note cards when speaking with the "parent." Some described their interpersonal skills as awkward as a result of pretending their classmate was a parent. This theme was represented in the following quote:

I think it was good that I addressed her as "Mrs. Smith" and I asked "How are you?" However, upon initiation of the interview, I should have shook her hand and introduced myself as the speech-language pathologist who will be providing services to Johnny.

Another participant contributed to this theme in the following statement: "I think because I felt nervous I was rambling and the conversation didn't flow as I had hoped... Overall, I thought my interpersonal skills were decent, but not a performance I would hope for."

\section{Major theme 2: Clinical conversations}

The second major theme revolved around the topic of clinical conversations with the classmate. This theme describes the different ways in which participants described the nature of stuttering, diagnostic interpretations, and treatment recommendations which all revolved around clinical conversations. These descriptions included explaining the definition of stuttering, characteristics of stuttering, and management strategies.

Minor theme 3: Improvements with explaining stuttering revealed This minor theme referred to the participants' reflections on areas of improvement for explaining stuttering, such as knowing factual information, and conveying information in a jargonfree manner. Furthermore, this theme represented areas in which participants could have "done better." For example, the following narrative response from one participant represents this theme: “...I feel that parents of children who stutter want an exact explanation of the cause of stuttering. This is a hard topic to address since an exact cause cannot be determined. I could have improved by ensuring the parent that it is not her fault."

Minor theme 4: Effective explanations

This theme refers the participants' perceptions and comfort in providing accurate information and effective explanations to the parent. Student reports indicated concise statements related to feeling like they did a "good job" with explaining certain aspects of stuttering. As one student put it: "I was very comfortable with discussing the nature and incidence of stuttering in the mock interview." In addition, another participant shared this experience when they concisely wrote "I think I explained the incidence and prevalence in a parent-friendly manner."

\section{Major theme 3: Impact of the experience}

This theme describes different ways in which participants reflected on the impact of the experience with a peer acting as a parent. The participants commented on various ways the assignment was beneficial to their learning. In addition, participants commented on how strengths and weaknesses were revealed as a result of the experience.

Minor theme 5: Beneficial learning experience

This theme refers to the participants' comments on ways that they benefitted from the assignment through learning, or how the exercise was helpful to their learning. Furthermore, participants commented on how effective they thought the interaction was with their classmate. For instance, some participants described the exercise as a helpful prelude to working with a real parent, as one participant wrote:

I think this experience really helped me learn the material. It also helped prepare me for when I will be doing this in real life. The assignment provided a good outline for how I should present this information in real life.

Minor theme 6: Strengths and weaknesses revealed

This theme refers to participant comments on personal 
strengths and weaknesses that were revealed during the interaction. These new areas consisted of addressing questions where participants were unsure of the answer, speaking with an emotional parent, and identifying areas where more of their knowledge was needed to answer questions. The following statement provided by one of the participants represents this theme:

This definitely made me realize that meetings like that need to be will organized and well researched. I felt nervous and scattered and I wasn't even talking with a real client's mother. I can only imagine how much more nervous I would be giving a diagnostic meeting.

\section{Actor experience reflections}

Thematic analysis of the actor experiences revealed six minor themes, which contributed to the formation of three major themes. These major themes consisted of reflections on interpersonal skills, clinical conversations, and the benefit of the experience with actors. Each major and minor theme will be discussed individually in the following section.

\section{Major theme 1: Interpersonal skills}

This first major theme described the different ways in which participants reflected on their interactions with an actor portraying the role of a parent. Topics related to this major theme included reflections on use of eye contact, how empathy was being conveyed, and responding appropriately to parent emotions. This theme was further sub-divided into two minor themes, which are described below.

Minor theme 1: Eye contact was good or needed improvement The first minor theme which contributed to major theme 1 included responses related to eye contact, with participants indicating eye contact was "better" or making some comment related to the idea of "improvement" with eye contact. For example, one participant wrote the following: "I feel like I could have maintained better eye contact. When I would get stuck on how to answer a question, I tended to look away instead of maintain full eye contact with her." Another participant shared their experience was better than the first with the classmate when they wrote "I feel as though my ability to maintain eye contact during the conversation was better compared to the prior experience with a classmate."

\section{Minor theme 2: Effective interpersonal skills}

For this second minor theme, participants made general, matter-of-fact statements related to how they felt they did a "good job" with using appropriate interpersonal skills, such as introducing themselves and using appropriate professional interpersonal skills. For example, one student wrote, "I feel that I had good interpersonal skills while talking with the student actor parent. The mother was very concerned and worried so I think I responded to her in a way that showed empathy." Another shared this feeling of using appropriate interpersonal skills in their comment "I think my interpersonal skills were appropriate during the interaction with the student actor. I introduced myself and used correct grammar \& speech."

\section{Major theme 2: Clinical conversations}

This second major theme described the different ways in which participants reflected on their ability to explain aspects of stuttering to the actor. For example, some of the reflections revolved around participants' ability to discuss the nature of stuttering, diagnostic interpretations, and treatment recommendations. This theme was further sub-divided into two minor themes.

Minor theme 3: Effective communication about nature of stuttering In this minor theme, participants reported feeling effective in their explanations related to discussing stuttering, or therapeutic procedures used. Furthermore, some participants provided comments related to improvements in this area because it was the second time around. For example, one participant wrote "I began the session discussing the overall nature of stuttering relevant to the client's case history. I feel like I explained the prevalence, incidence, ratio, etc. in a parent friendly way." Furthermore, another student commented the following: "I feel like I appropriately answered the questions regarding the nature of stuttering in a way in which the parent would understand."

Minor theme 4: Areas of improvement with clinical discussions The fourth minor theme revealed areas of improvement with the clinical interaction. This minor theme involved student stating that some of explanations with the parent lacked detail and participants commented that more information could have been included. Some of these responses were relatively brief, with participants writing statements conveying the sentiment of "I could have" done something different reflecting back. For example, one participants communicates this area of improvement in the following reflection: 
I feel as though it improved from the prior experience with a classmate but could still use some improving. I believe I touched on all the components for the assignment, but did so rather briefly. When preparing for the assignment I planned on defining/expanding upon concepts I brought up. However, after much thought I decided against it as I thought it may be "too much" information (in a real life situation). As a parent I would want to know only information related to my child, especially when being faced with given a diagnosis of any sort.

In addition, another student conveyed an area of improvement in the following response: "Also, I have confidence that I effectively answered the mother's questions related to the nature of stuttering. However, I should have provided more details and explanation for the variability of stuttering."

\section{Major theme 3: Impact of experience}

This final major theme described the different ways in which participants reflected on the impact of the experience with an actor acting as a parent. This theme reflected participant comments related to how genuine the experience was with the actor, as well as new insights, which were gained through interacting with the actor. This theme was further sub-divided into two minor themes.

\section{Minor theme 5: Natural flow, real-world, and genuine}

This theme reflected participants statements related to the genuineness, or "real world" feelings of the activity. Furthermore, participants commented that the experience flowed more naturally and seemed more practical because the actor felt like an "actual" or "real" person. Thus, the interaction felt more genuine and "real-life." One participant communicated their satisfaction with the experience compared to their classmate and this "real world" feeling gained where they wrote:

I actually enjoyed role playing with the student actor more so than my classmate. It was very natural, and provided me with an idea of what to expect in the real world. It also helped me to understand how important it will be to empathize with parents, but still conveying what I need to say.

Another example of the genuine feeling conveyed during the experience is reflected in another statement by a student where they commented on how "stressed" the actor felt during the interaction:
I think this experience was much more beneficial than the student-to-student interaction. I felt like I was dealing with an actual client. The actress seemed genuinely stressed and concerned which caused me to add empathy and detail to my responses.

\section{Minor theme 6: New insights}

This minor theme consisted of participant comments in regard to insights gained through the experience. Participant stated that they were made more aware of the process of their clinical skills related to an evaluation, or the experience made them realize something new. For example, one participant wrote:

This experience with the simulated actor has greatly impacted my learning in a positive manner. It has made me more aware of my strengths and weaknesses. Now, I know how to fix my mistakes and therefore, I'm a better clinician because of it.

Another participant indicated their insight about how they need to not have preconceived notions regarding how a clinical situation will unfold where they wrote:

I learned not to have certain ideas about how the interview will go. I tried to anticipate and prepare for questions that weren't asked. I learned that I needed to have an open mind about the situation and the feelings and questions the parent might have.

\section{DISCUSSION AND CONCLUSIONS}

The results from this study are promising in that they provide support for the use of trained actors in a graduate fluency disorders course. Participant experiences with trained actors revealed reports of new insights in their clinical abilities, which were not as present in their interactions with peers. Although interacting with a peer was found to be helpful, the inclusion of actors brought the experience to a different level for students. It could be said that the interaction with the actor allowed for the students to appreciate the complexities of communicating information about stuttering in a more authentic clinical situation. Participants also perceived their learning experience with actors as memorable and worthwhile because of the "real world" feelings that the actor assignment invoked. Because of the perceived practicality of this assign- 
ment, it reportedly felt "real" to the students and it allowed them the opportunity to better understand aspects of the course that directly related to stuttering.

These benefits, that came to be as a result of purposefully incorporating trained actors in a graduate-level fluency disorders course, are similar to findings uncovered by Steyl et al. [22]. Steyl et al. [22] spoke about the importance of incorporating experiential-based learning opportunities within a graduate-level fluency disorders course. This approach to education focuses more on designing and implementing learning activities that allow students to be active participants within the classroom setting, as opposed to just passively consuming content through textbooks and lectures. Specifically, Steyl and colleagues [22] discovered positive findings when graduate students were presented with active learning opportunities that had an Internet component to them, such as watching a number of pre-recorded teletherapy videos of adults who stutter and then using an Internet-based videoconferencing software to interview those clients. From being able to interact with people who stutter through the Internet, a significant increase was noted in the graduate students' perceived knowledge and comfort level, with regards to stuttering. This increase was apparent in large part due to the fact that their fluency disorders course, like the course highlighted in the present study, allowed graduate students to experience course content.

These findings are also similar with Syder's [12] conclusions regarding the use of simulated clients. In Syder's study [12], first and second year students were included among the three groups and their concluding remarks highlighted that participants felt the experience was generally beneficial. The current study's methodology differed with that of Syders, in that an actor was used as a parent in the current study, whereas an actor was taking on the role of a client in Syder's study. In their recommendations, Syder [12] indicated that actors could be used throughout a graduate student's career.

\section{Limitations}

Although the current findings provide valuable information regarding the benefit of using actors in the clinical education of stuttering, several important limitations need to be acknowledged in light of the results. First, the current study did not provide a formal procedure to determine the believability of actor portrayals. More recently, Baylor et al. [11] provided a formal procedure to determine how believable standardized patients were in taking on the role of a person with aphasia and dysarthria, and found that actors were found to be believable in their portrayals. Although the present study did not incorporate a formal procedure for believability, one of the themes related to the students' experience with the actor was the genuine portrayal of the actor during their interaction. Nevertheless, there was not a quantitative rating scale that was used to determine the extent to which participants believed the person was believable on certain areas. Future studies may wish to incorporate the procedures of Baylor et al. [11] to measure believability of the portrayal.

Second, the familiarity with the simulated clinical conversation could have potentially contributed to increased confidence in interacting with the actor. Two areas could have contributed to this limitation and consisted of student preparation for the interaction with the peer and actor, as the time in which students prepared for the conversation was not controlled. Also, bias may have been interjected into the study by providing students with the general outline of the conversation prior to the interaction. With this bias, students may have been able to rehearse their interactions more, thus, decreasing the spontaneity of the interaction with the peer and actor. Another area that contributed to this limitation was the fact that students interacted with the actor during the second situation. Therefore, the increased confidence may have been related to the fact that this experience was their second time discussing the hypothetical case and not because of the actors.

A third limitation was related to a lack of control regarding which student was assigned to one of the two actors. Recall that both actors had different biographical sketches, with one actor portraying a parent who was overly concerned and the other portraying a "disengaged" parent. These actor differences could have contributed to students' self-perceptions of clinical skills. Furthermore, the data was analyzed consistent with the purpose of the study, meaning that it was analyzed based upon the actor and student experience and not the specific actor. As a result, these findings are unable to compare participant findings between the two actors, as the data was not analyzed in this manner due to the preliminary nature and purpose of the study.

Another limitation was related to data collection. In regard to the qualitative data, participant narrative responses varied in length, with some responses being very descriptive and others being relatively brief and to the point (i.e., 1-2 sentences). With the nature of these data, the researchers were unable to request additional information from participants. With these short responses, it was difficult to obtain the depth 
of understanding from the qualitative responses. For the quantitative data, student ratings for both the SCS-P and SCSA were not formally validated through another rater. The inclusion of an outside rater to determine if participants' selfassessments were reliable and accurate would have helped to validate the findings.

Finally, due to the logistics of the stuttering course, we were not able to create a control group. Ideally, it would have been beneficial to have half the class interact with a classmate, then the other half meet with the actor. Perceptions could then be compared and more clarity could be provided regarding the effect of actors on student learning. Future studies examining the benefit of actors in clinical education are encouraged to incorporate a control group.

\section{Clinical education implications}

Despite these limitations, the present study's findings have implications for the clinical education of speech-language pathology students related to stuttering. Although previous simulation studies have reported clinician-client interactions $[10,12]$, these findings represent one of the first studies to report on simulated clinician-parent interactions. There has been a consistent finding throughout the years that more clinical training is needed in the area of stuttering as many clinicians have reported feeling unprepared to work clinically with stuttering [23,24]. The use of actors in the clinical training of stuttering could provide another way to educate future generations of speech-language pathologists to feel more confident when working with individuals who stutter and their families.

In addition, there was much overlap in terms of findings related to actor and peer experiences, however, subtle differences existed between the two experiences. For example, the actor experience revealed more of a genuine experience and generated new insights with students compared to the peer experiences. It appears as if interacting with the actor allowed the students to become more aware about possibilities that can occur during a clinical interaction that a peer could not provide, perhaps due to the familiarity of the classmates and the predictability which may accompany a scripted conversation with a classmate.

Although preliminary in nature, this study attempts to provide a description for how actors can be incorporated in a graduate fluency disorders course. Results suggest that including trained actors in the clinical education of speech-language pathology master's students was beneficial to their clinical skill development in communicating with a hypothet- ical parent of a preschooler with speech fluency concerns. Despite the limitations, these findings are promising, showing that actors can greatly contribute and enhance clinical education in stuttering.

\section{ACKNOWLEDGMENTS}

Actors were compensated in whole through the UniversityFunded Scholarship Program at Eastern Kentucky University. Thank you to Charity Yarzebinski for her assistance. Also, thank you to Dr. Jerry Hoepner for his editorial feedback. The current study's findings were partially presented as a poster presentation at the 2015 Annual Convention of the American Speech-Language-Hearing Association. Finally, thank you to the participants who took part in this study.

\section{REFERENCES}

1. Haracz K, Arrighi G, Joyce B. Simulated patients in a mental health occupational therapy course: a pilot study. British Journal of Occupational Therapy. 2015;78:757-766.

2. Kassab ES, King D, Hull LM, Arora S, Sevdalis N, Kneebone RL, et al. Actor training for surgical team simulations. Medical Teacher. 2010;32:256-258.

3. Levitov JE, Fall KA, Jennings MC. Counselor clinical training with client-actors. Counselor Education \& Supervision. 1999;38:249259.

4. Paparella-Pitzel S, Edmond S, DeCaro C. The use of standardized patients in physical therapist education programs. Journal of Physical Therapy Education. 2009;23:15-23.

5. Gregg BA. Initial counseling with parents of preschoolers who stutter: enhancing graduate students' skills using simulated caregivers. Perspectives on Fluency and Fluency Disorders. 2013;23:2129.

6. Zraick R. The use of standardized patients in speech-language pathology. Perspectives on Issues in Higher Education. 2002;5:14-16.

7. Zraick RI. Playacting with a purpose: using standardized patients to assess clinical skills. The ASHA Leader. 2004;9:22.

8. Zraick RI. Review of the use of standardized patients in speechlanguage pathology clinical education. International Journal of Therapy and Rehabilitation. 2012;19:112-118.

9. Hill AE, Davidson BJ, Theodoros DG. A review of standardized patients in clinical education: implications for speech-language pathology programs. International Journal of Speech-Language Pathology. 2010;12:259-270.

10. Zraick RI, Allen RM, Johnson SB. The use of standardized patients to teach and test interpersonal and communication skills with students in speech-language pathology. Advances in Health Sciences Education. 2003;8:237-248.

11. Baylor C, Burns MI, Struijk J, Herron L, Mach H, Yorkston K. As- 
sessing the believability of standardized patients trained to portray communication disorders. American Journal of Speech-Language Pathology. 2017;26:791-805.

12. Syder D. The use of simulated clients to develop the clinical skills of speech and language therapy students. European Journal of Disorders of Communication. 1996;31:181-192.

13. Guitar B. Stuttering: An Integrated Approach to Its Nature and Treatment (4th ed). Baltimore: Lippincott Williams \& Wilkins, 2013.

14. Benedetti RL. The actor in you: sixteen simple steps to understanding the art of acting. Boston: Allyn \& Bacon; 2012.

15. Chekhov Association (n.d.). Retrieved May 8, 2017, from http:// www.michaelchekhov.org/

16. Hagen U. A challenge for the actor. New York: Scribner, 1991.

17. Zebrowski PM, Schum RL. Counseling parents of children who stutter. American Journal of Speech-Language Pathology. 1993;2: 65-73.

18. Guitar B, Guitar C, Fraser J, Chmela KA, Hill DG, Ramig PR, et al. [producers]. (2006). Stuttering and Your Child: Help for Parents [video]. The Stuttering Foundation. Available from: https://www. youtube.com/watch?v=u2_mgt87g1Y
19. Spolin V. Improvisation for the theater a handbook of teaching and directing techniques. Evanston, IL: Northwestern University Press, 1999.

20. Creswell JW. Qualitative inquiry and research design: choosing among five approaches (2nd Ed.). Thousand Oaks, CA: Sage Publications; 2007.

21. Hughes CD, Gabel R, Daniels DE. Discussing stuttering with parents: a preliminary study of the experiences of adolescents who stutter. Speech, Language, and Hearing. 2015;18:44-54.

22. Steyl NA, Klein JF, Howell ML, Dalton JC. Increasing knowledge and skills of graduate students in a fluency disorders course. Contemporary Issues in Communication Science and Disorders. 2016;43:164-173.

23. Brisk DJ, Healey EC, Hux KA. Clinicians' training and confidence associated with treating school-age children who stutter: a national survey. Language, Speech, and Hearing Services in Schools. 1997;28:164-176.

24. Mallard AR, Gardner LS, Downey CS. Clinical training in stuttering for school clinicians. Journal of Fluency Disorders. 1998; 13:253-259. 


\section{APPENDIX A. Directions for open-ended responses}

\section{Directions}

Reflect upon your experience during your experience as the clinician during the role play exercise with your classmate. Provide a written reflection to each of the three areas: Interpersonal Skills, Nature of Stuttering, and Diagnostic Interpretation and Recommendations. Finally, provide your thoughts about the impact this particular part of the assignment had on your learning. When responding to each section, make sure to reflect upon your strengths, areas of improvement, and any other observations you would like to report.

Interpersonal Skills

Nature of Stuttering

Diagnostic Interpretation and Recommendations

Describe the impact this experience with role playing with a classmate had on your learning. 